\title{
Estudo da relação estrutura-atividade de saponinas hemolíticas e/ou imunoadjuvantes mediante uso de análise multivariada
}

\author{
Samuel Kaiser, Cabral Pavei, George G. Ortega*
}

Faculdade de Farmácia, Universidade Federal do Rio Grande do Sul, Laboratório de Desenvolvimento Galênico, Av. Ipiranga, 2752, 90610-000 Porto Alegre-RS, Brasil.

\begin{abstract}
RESUMO: Entre as diversas atividades biológicas relatadas para as saponinas, merecem destaque aquelas relacionadas ao aumento da resposta imune e a ruptura das membranas dos eritrócitos. No desenvolvimento de vacinas, ambas as propriedades exercem atividades antagônicas, contudo, as informações sobre as relações estrutura-atividade são relativamente escassas e, às vezes, conflitantes. O presente trabalho visa contribuir no estabelecimento das relações estruturais envolvidas com as atividades imunoadjuvante e hemolítica de saponinas triterpênicas. Para isso, foram selecionadas vinte saponinas de estrutura triterpênica, isoladas das espécies Aesculus hippocastanum, Dolichos lablab e Glycine max. A relação entre grupamentos substituintes do anel triterpênico e as atividades biológicas foi estudada mediante análise de agrupamentos e análise de componentes principais. Os resultados confirmam a importância da presença de açúcares em C-3 para a atividade hemolítica. Porém o efeito causado pela presença de uma hidroxila em $\mathrm{C}-16$, de $\mathrm{CH}_{2} \mathrm{OH}$ em C-17, de uma acetila em C-22 e de um grupamento acila em C-21 sobre essa atividade parece ser mais acentuado. Já a presença de uma hidroxila em C-21, de uma metila em C-17 e de dois açúcares ligados à aglicona parece ser determinante para a atividade imunoadjuvante. Além disso, observa-se a existência de uma relação inversa entre as atividades hemolítica e imunoadjuvante.
\end{abstract}

Unitermos: Saponinas, relação estrutura-atividade, atividade imunoadjuvante, atividade hemolítica, análise de agrupamentos, análise de componentes principais.

\begin{abstract}
Study of the structure-activity relationship of adjuvant and/or hemolytic saponins by use of multivariate analysis”. Among the various biological activities reported for saponins, those related with increase immune response and the destruction of the membrane of red blood cells deserve attention. In the development of vaccines, these properties exert antagonistic activities. However, the information about the structure-activity relationships is relatively scarce and sometimes contradictory. This paper aims to contribute to the establishment of structural relations involved with the hemolytic and adjuvant activities of triterpenic saponins by the analysis of the different groups linked to aglycone, with the use of multivariate statistical techniques. For this, we had twenty triterpenic saponins previously isolated from Aesculus hippocastanum, Dolichos lablab. and Glycine max species. Their adjuvant and hemolytic activities are available in the literature. These saponins were analyzed as groups linked to the triterpenic ring and biological activities resulting in an array of date that was submitted to cluster analysis and principal components analysis. The results confirm the importance of sugars in $\mathrm{C}-3$ for hemolytic activity. However, the effect caused by the presence of a hydroxyl in $\mathrm{C}-16, \mathrm{CH}_{2} \mathrm{OH}$ in $\mathrm{C}-17$, acetyl in $\mathrm{C}-22$ and acyl in $\mathrm{C}-21$ on the hemolytic activity seems to be more important. On the other way, the presence of a hydroxyl in C-21, a methyl in C-17 and two sugars linked to aglycone seem determinant for adjuvant activity. Moreover, there is an inverse relationship between adjuvant and hemolytic activities in the set of saponins analyzed.
\end{abstract}

Keywords: Saponins, structure-activity relationship, adjuvant activity, hemolytic activity, cluster analysis, principal components analysis.

\section{INTRODUÇÃO}

Dentre os metabólitos secundários produzidos pelos vegetais, as saponinas constituem uma das classes de maior destaque devido à sua ampla distribuição no reino vegetal e suas importantes atividades biológicas
(Schenkel et al., 2003). Com o surgimento de novas gerações de vacinas, principalmente aquelas baseadas em proteínas recombinantes e DNA, que são menos reativas e imunogênicas se comparadas às vacinas tradicionais, o potencial imunoadjuvante e hemolítico desses compostos tem chamado a atenção (O’Hagan et al., 2001). 
Alguns pesquisadores reconhecem a possibilidade da atividade imunoadjuvante estar relacionada com o tamanho e a natureza das cadeias laterais osídicas (Kensil et al., 1992), contudo, as evidências experimentais a esse respeito ainda são insuficientes. Além disso, muitas informações relacionadas à atividade imunoadjuvante se referem especificamente às saponinas de Quillaja ou seus derivados semi-sintéticos (Soltysik et al., 1995; Kensil et al., 1996), os quais, embora apresentem uma excelente atividade imunoadjuvante, são estruturalmente diferentes de outras saponinas com atividade imunoadjuvante descrita.

Vários trabalhos foram realizados na busca de saponinas com alta capacidade imunogênica e baixa capacidade hemolítica (Estrada et al., 2000; Sun et al., 2005; Fleck et al., 2006; Sun, 2006; Sun \& Pan, 2006). Contudo, em nenhum desses trabalhos é exposta uma relação estrutura-atividade clara para esta classe de compostos, o que se deve, principalmente, a ausência de trabalhos envolvendo a sistematização das informações disponíveis.

A análise de componentes principais é um método estatístico de análise multivariada que permite estabelecer correlações entre as diversas variáveis que fazem parte de uma matriz complexa, propiciando o estabelecimento de relações entre as mesmas que, de outro modo, permanecem ocultas. Já a análise de agrupamentos ou de cluster, que também faz parte da análise multivariada, permite agrupar objetos heterogêneos segundo determinadas características, de modo a construir classes distintas, mas de elevada homogeneidade interna (Hair et al., 2005).

O presente trabalho visa contribuir no estabelecimento das relações estruturais envolvidas com as atividades imunoadjuvante e hemolítica de saponinas triterpênicas, mediante uma análise dos diferentes grupamentos ligados à aglicona via técnicas estatísticas multivariadas.

\section{MATERIAIS E MÉTODOS}

\section{Seleção das saponinas}

Foram selecionadas vinte saponinas de estrutura triterpênica do conjunto de saponinas estudadas por Oda et al. (2000), isoladas das espécies Aesculus hippocastanum L. (castanha-da-india), Dolichos lablab L. (lablab) e Glycine max L. (soja).

Avaliação dos grupamentos substituintes e atividades biológicas

Foram avaliados os substituintes e os açúcares ligados à aglicona nas posições C-3, C-4, C-16, C-17, C-21, C-22, C-28, utilizando o sistema de codificação por variáveis binárias (1 indica presença e 0 ausência de determinado grupamento substituinte ou açúcar na aglicona). Os resultados referentes às atividades imunoadjuvante e hemolítica das saponinas foram extraídos do estudo realizado por Oda et al. (2000) e expressas pelo Título de Hemaglutinação Passiva (PHA) e pela Concentração Hemolítica 50\% (CH50\%), respectivamente. Estas variáveis numéricas foram transformadas segundo uma escala de 0,5 a 4,5 , para $\mathrm{PHA}$, e de 0,5 a 2 para $\mathrm{CH} 50 \%$.

\section{Análise de agrupamentos}

O procedimento hierárquico aglomerativo utilizado foi o de ligação individual e as medidas utilizadas foram do tipo correlacionadas (correlação dos coeficientes de distância). O número de agrupamentos extraídos foi direcionado pela diferença entre o nível de similaridade do novo agrupamento formado a cada passo do procedimento hierárquico, em relação ao anterior. Foram realizadas análises da matriz de origem e da matriz transposta, na presença de atividades biológicas (PHA e $\mathrm{CH} 50 \%$ ) e na ausência destas.

\section{Análise de componentes principais}

O número de componentes extraídos foi determinado mediante o critério da raiz latente. Para isso foram considerados significativos apenas os componentes com raízes latentes ou autovalores maiores que a unidade (Hair et al., 2005). Foram realizadas análises da matriz de origem e da matriz transposta, na presença de atividades biológicas (PHA e $\mathrm{CH} 50 \%$ ) e na ausência destas, assim como na análise de agrupamentos.

Todas as análises estatísticas aplicadas neste trabalho foram realizadas com o auxílio do programa estatístico Minitab 14® (Minitab Inc., 2005).

\section{RESULTADOS E DISCUSSÃO}

\section{Seleção das saponinas}

O efeito hemolítico das saponinas é fortemente influenciado pela natureza polar dos substituintes ligados à aglicona (Voutquenne et al., 2002), da mesma forma que a atividade imunoadjuvante (Soltysik et al., 1995; Kensil et al., 1996), criando um universo amplo de possíveis relações estrutura-atividade. Por esse motivo, no presente trabalho foram selecionadas 20 saponinas triterpênicas pentacíclicas (Tabela 1) derivadas de ácido oleanólico, sojassapogenóis $\mathrm{A}$ e $\mathrm{B}$, hederagenina, protoaescigenina e barringtogenol $\mathrm{C}$, com as mesmas configurações em C-3, C-4, C-8, C-10, C-14, C-17 e C-20. Estas saponinas pertencem aos grupos das sojassaponinas, dos lablabosídeos e das escinas, isoladas de G. max, D. lablab e A. hippocastanum, respectivamente.

O grupo selecionado visou incluir saponinas com atividade hemolítica e imunoadjuvante, com atividade 
Tabela 1. Representação estrutural das saponinas triterpênicas derivadas de (A) G. max (B) D. lablab e (C) A. hippocastanum.

\begin{tabular}{|c|c|c|c|c|}
\hline Estrutura & Saponina (Abreviação) & $\mathrm{R}_{1}$ & $\mathrm{R}_{2}$ & $\mathrm{R}_{3}$ \\
\hline & $\begin{array}{l}\text { Sojassapogenol A } \\
\text { (SYOL - A) }\end{array}$ & $\mathrm{OH}$ & $\mathrm{H}$ & $\mathrm{H}$ \\
\hline & $\begin{array}{l}\text { Sojassapogenol B } \\
\text { (SYOL - B) }\end{array}$ & $\mathrm{H}$ & $\mathrm{H}$ & $\mathrm{H}$ \\
\hline & $\begin{array}{l}\text { Sojassaponina I } \\
\quad(\text { SYA - 1) }\end{array}$ & $\mathrm{H}$ & $\mathrm{H}$ & -AcGlc-Gal-Rha \\
\hline & $\begin{array}{c}\text { Sojassaponina II } \\
\text { (SYA - 2) }\end{array}$ & $\mathrm{H}$ & $\mathrm{H}$ & -AcGlc-Ara-Rha \\
\hline & $\begin{array}{c}\text { Sojassaponina III } \\
\text { (SYA - 3) }\end{array}$ & $\mathrm{H}$ & $\mathrm{H}$ & -AcGlc-Gal \\
\hline 3 & $\begin{array}{c}\text { Deidrosojassaponina I } \\
\text { (DHSYA ) }\end{array}$ & $\mathrm{H}$ & $* * *$ & -AcGlc-Gal-Rha \\
\hline 24 & $\begin{array}{l}\text { Sojassapogenol A } \\
\text { (SYOL - A) }\end{array}$ & $\mathrm{OH}$ & $\mathrm{H}$ & $\mathrm{H}$ \\
\hline A & $\begin{array}{l}\text { Sojassapogenol B } \\
\text { (SYOL - B) }\end{array}$ & $\mathrm{H}$ & $\mathrm{H}$ & $\mathrm{H}$ \\
\hline & $\begin{array}{l}\text { Lablabosideo A } \\
\quad(\text { LBL - A) }\end{array}$ & $\mathrm{H}$ & Glc & $\#$ \\
\hline & $\begin{array}{l}\text { Lablabosideo B } \\
\text { (LBL - B) }\end{array}$ & $\mathrm{OH}$ & Glc & \# \\
\hline & $\begin{array}{l}\text { Lablabosideo C } \\
\quad(\text { LBL - C) }\end{array}$ & $\mathrm{OH}$ & -Glc-Rha & \# \\
\hline $\begin{array}{l}\mathrm{AcGlc} \\
\mathrm{I} \\
\mathrm{Gal}\end{array}$ & $\begin{array}{l}\text { Lablabosideo D } \\
\text { (LBL - D) }\end{array}$ & $\mathrm{OH}$ & -Glc-Hmg & \# \\
\hline Rha & $\begin{array}{l}\text { Lablabosideo E } \\
\quad(\text { LBL - E) }\end{array}$ & $\mathrm{OH}$ & - Glc-Rha-Rha & $\#$ \\
\hline B & $\begin{array}{l}\text { Lablabosideo F } \\
\quad(\text { LBL - F })\end{array}$ & $\mathrm{H}$ & -Glc-Rha-Rha & \# \\
\hline \multirow[b]{6}{*}{$\mathrm{G}$} & $\begin{array}{l}\text { Escina Ia } \\
(\mathrm{ESC}-1 \mathrm{~A})\end{array}$ & Tig & $\mathrm{OH}$ & Glc \\
\hline & $\begin{array}{l}\text { Escina Ib } \\
(\mathrm{ESC}-1 \mathrm{~B})\end{array}$ & Ang & $\mathrm{OH}$ & Glc \\
\hline & $\begin{array}{c}\text { Escina IIa } \\
(\text { ESC - 2A) }\end{array}$ & Tig & $\mathrm{OH}$ & Xyl \\
\hline & $\begin{array}{l}\text { Escina IIb } \\
(\text { ESC - 2B) }\end{array}$ & Ang & $\mathrm{OH}$ & Xyl \\
\hline & $\begin{array}{l}\text { Escina IIIa } \\
(\mathrm{ESC}-3 \mathrm{~A})\end{array}$ & Tig & $\mathrm{H}$ & Glc \\
\hline & $\begin{array}{l}\text { Escina IIIb } \\
(\mathrm{ESC}-3 \mathrm{~B})\end{array}$ & Ang & $\mathrm{H}$ & Glc \\
\hline
\end{tabular}

*** Deidrossojassaponina I apresenta uma carbonila no carbono 22 e portanto não apresenta substituinte em $\mathrm{R}_{2}$;

\# ausência de $\mathrm{R}_{3}$.

Monômeros presentes na cadeia de açúcares: Ácido Glicurônico (AcGlc), Arabinose (Ara), Glicose (Glc), Galactose (Gal), Ramnose (Rha) e Xilose (Xyl);

Grupamentos acila presentes: 3-hidroxi-3-metilglutaroil (Hmg), Angeloil (Ang) e Tigloil (Tig). 
Tabela 2. Grupamentos substituintes e abreviações das variáveis utilizadas na análise estrutural das saponinas triterpênicas.

\begin{tabular}{|c|c|c|}
\hline $\begin{array}{c}\text { Local de } \\
\text { substituição }\end{array}$ & Grupamento substituinte & Abreviação \\
\hline \multirow{8}{*}{ Carbono 3 (C-3) } & Hidroxila $(\mathrm{OH})$ & $\mathrm{C} 3-\mathrm{OH}$ \\
\hline & Gal $(1 \rightarrow 2)$ AcGlc - & $\mathrm{C} 3-\mathrm{a}$ \\
\hline & $\operatorname{Rha}(1 \rightarrow 2) \operatorname{Gal}(1 \rightarrow 2)$ AcGlc - & C3-b \\
\hline & Rha $(1 \rightarrow 2) \operatorname{Ara}(1 \rightarrow 2)$ AcGlc - & $\mathrm{C} 3-\mathrm{c}$ \\
\hline & $\operatorname{Gal}(1 \rightarrow 2) \operatorname{Rha}(1 \rightarrow 2)$ AcGlc - & C3-d \\
\hline & $\operatorname{Glc}(1 \rightarrow 2) \operatorname{Gal}(1 \rightarrow 2) A c G l c-$ & C3-e \\
\hline & $\operatorname{Glc}(1 \rightarrow 4) \operatorname{AcGlc}(2 \rightarrow 1) X y l-$ & $\mathrm{C} 3-\mathrm{f}$ \\
\hline & $\operatorname{Glc}(1 \rightarrow 4) \operatorname{AcGlc}(2 \rightarrow 1) \mathrm{Glc}-$ & C3-g \\
\hline \multirow{3}{*}{ Carbono 4 (C-4) } & Uma metila $\left(\mathrm{CH}_{3}\right)$ & C4-met \\
\hline & Duas metilas $\left(\mathrm{CH}_{3}\right)$ & C4-2met \\
\hline & $\mathrm{CH}_{2} \mathrm{OH}$ & C4-metox \\
\hline Carbono 16 (C-16) & Hidroxila $(\mathrm{OH})$ & $\mathrm{C} 16-\mathrm{OH}$ \\
\hline \multirow{3}{*}{ Carbono 17 (C-17) } & Metila $\left(\mathrm{CH}_{3}\right)$ & C17-met \\
\hline & $\mathrm{CH}_{2} \mathrm{OH}$ & C17-metox \\
\hline & COO-ligado a um açúcar & C17-carbose \\
\hline \multirow{3}{*}{ Carbono 21 (C-21) } & Hidroxila $(\mathrm{OH})$ & $\mathrm{C} 21-\mathrm{OH}$ \\
\hline & Tigloil & C21-tig \\
\hline & Angeloil & C21-ang \\
\hline \multirow{4}{*}{ Carbono 22 (C-22) } & Hidroxila $(\mathrm{OH})$ & $\mathrm{C} 22-\mathrm{OH}$ \\
\hline & Acetil (OCOCH3) & C22-carbmet \\
\hline & Carbonila & $\mathrm{C} 22$-carb \\
\hline & Glc $(1 \rightarrow 3)$ Ara - & $\mathrm{C} 22-\mathrm{a}$ \\
\hline \multirow{5}{*}{ Carbono 28 (C-28) } & Hidroxila $(\mathrm{OH})$ & $\mathrm{C} 28-\mathrm{OH}$ \\
\hline & Glc - & $\mathrm{C} 28-\mathrm{a}$ \\
\hline & Rha $(1 \rightarrow 4)$ Glc - & $\mathrm{C} 28-\mathrm{b}$ \\
\hline & $\operatorname{Hmg}(1 \rightarrow 6) \mathrm{Glc}-$ & $\mathrm{C} 28-\mathrm{c}$ \\
\hline & Rha $(1 \rightarrow 4)$ Rha $(1 \rightarrow 2)$ Glc - & $\mathrm{C} 28-\mathrm{d}$ \\
\hline
\end{tabular}

hemolítica e sem atividade imunoadjuvante e vice-versa, e aquelas que não apresentam nenhuma dessas atividades. A centralização sobre um mesmo trabalho como fonte de dados experimentais (Oda et al., 2000) permite comparar, de forma inequívoca, atividades imunoadjuvante e hemolítica determinadas por uma mesma metodologia, evitando, dessa maneira, introduzir viés nas análises.

\section{Avaliação dos grupamentos substituintes e atividades biológicas}

Os grupamentos substituintes associados às saponinas selecionadas encontram-se discriminados na Tabela 2. A cada grupo foi conferido o mesmo grau de importância visando eliminar qualquer tendência subjetiva nas análises posteriores.

A transformação das variáveis numéricas em escalas específicas evita distorções causadas pela análise conjunta, em uma mesma matriz de dados, das variáveis binárias e daquelas numéricas, originalmente relatadas por Oda et al. (2000) para PHA e CH50\%. Dessa forma, os resultados das codificações dos diferentes grupamentos substituintes e das atividades biológicas geram uma matriz de dados (Tabela 3), utilizada nas análises posteriores.

\section{Análise de agrupamentos}

A principal característica da análise de agrupamentos é a sistematização das relações entre grupamentos substituintes e as atividades biológicas PHA e $\mathrm{CH} 50 \%$, fazendo com que cada agrupamento reúna características similares, agregando aspectos estruturais segundo a magnitude das atividades imunoadjuvante e hemolítica relatadas para cada saponina. Na figura 1 observam-se os diferentes dendrogramas obtidos a partir da análise de agrupamentos da matriz de origem (análise dos grupamentos substituintes) e da matriz transposta (análise das saponinas).

A matriz de origem corresponde aos dados representados na Tabela 3. Já a matriz transposta permite transformar as saponinas (originalmente ocupando as linhas) em variáveis da matriz, ao mesmo tempo em que os substituintes, que originalmente ocupavam as colunas, passam a constituir as linhas ou as observações da matriz.

A distinção entre as estruturas das saponinas selecionadas pode ser evidenciada melhor se desconsiderarmos inicialmente as atividades biológicas PHA e $\mathrm{CH} 50 \%$, o que resulta na formação de quatro agrupamentos distintos (Figura 1A). $\mathrm{O}$ primeiro agrupamento (saponinas de G. max) é composto por três subpopulações: a primeira inclui as agliconas SYOL-A e SYOL-B, a segunda as saponinas monodesmosídicas SYA-A, SYA-B e SYA-C, e a terceira as saponinas bidesmosídicas SYA-A1 e SYA-A2. Observa-se que a saponina DHSYA constitui um agrupamento próprio (terceiro agrupamento), fato que reflete suas características estruturais exclusivas, como a carbonila em C-22, que a diferencia das demais saponinas de soja. Os lablabosídeos que compõem o segundo agrupamento se distribuem em duas subpopulações: a primeira inclui LBL-A e LBL-F, que apresentam duas metilas em C-4, e a segunda é composta pelos demais lablabosídeos, que apresentam uma metila e um $\mathrm{CH}_{2} \mathrm{OH}$ em C-4. O quarto agrupamento compreende as saponinas de $A$. hippocastanum, que se distribuem em duas subpopulações: a primeira inclui ESC-3A e ESC$3 \mathrm{~B}$, que apresentam duas metilas em $\mathrm{C}-4$, e a segunda as demais escinas, que apresentam uma metila e um $\mathrm{CH}_{2} \mathrm{OH}$ em C-4.

Pode-se afirmar que o modelo adotado permite discriminar as saponinas segundo a espécie vegetal de origem. As diferentes subpopulações de lablabosídeos e escinas são definidas dentro de um mesmo agrupamento em virtude dos substituintes em C-4, o que não ocorre nas sojassaponinas, onde as diferentes subpopulações são determinadas pelo número de cadeias de açúcares ligados a aglicona, já que possuem o mesmo substituinte em C-4. A posição do ponto de bifurcação que liga uma subpopulação 
a outra em um mesmo agrupamento é, geralmente, determinada pela semelhança entre os açúcares ligados a aglicona.

A inserção das atividades biológicas PHA e $\mathrm{CH} 50 \%$ introduzem alterações claras no dendrograma (Figura 1B) demonstrando a influência de ambas variáveis no contexto geral das relações estruturais observadas na figura $1 \mathrm{~A}$. O agrupamento $1 \mathrm{G}$ discrimina as saponinas segundo uma alta capacidade imunoadjuvante e escassa capacidade hemolítica. A subpopulação formada pelas agliconas SYOL-A e SYOL-B fica segregada em função da sua baixa capacidade imunoadjuvante e hemolítica. A ausência de atividade hemolítica para saponinas derivadas dos sojassapogenóis $\mathrm{A}, \mathrm{B}$ e $\mathrm{F}$ foi relatada previamente (Gestetner et al., 1971; Oda et al., 2000; Voutquenne et al., 2002) e coincide com a estrutura observada no dendrograma. A ausência de atividade hemolítica em saponinas de soja, tanto mono- quanto bidesmosídicas, sugere que a natureza e o número de açúcares presentes nas cadeias glicosídicas estão subordinados às características estruturais da aglicona. As saponinas que apresentam um potencial imunoadjuvante moderado passam a compor o segundo agrupamento ( $2 \mathrm{G})$, enquanto o terceiro $(3 \mathrm{G})$ é composto por saponinas que apresentam elevado potencial hemolítico e baixo potencial imunoadjuvante.

Embora as saponinas monodesmosídicas glicosiladas em C-3 sejam frequentemente associadas a uma maior atividade hemolítica, se comparadas às bidesmosídicas com glicosilação em C-3 e C-28 (Hase et al., 1981; Voutquenne et al., 2002), isso não coincide com a fraca capacidade hemolítica constatada para as saponinas do agrupamento $1 \mathrm{G}$, que são em sua maioria monodesmosídicas glicosiladas em C-3. Isso reforça a influência decisiva da aglicona sobre a atividade hemolítica. Essa contradição aparente entre a natureza mono- e bidesmosídica e a capacidade hemolítica das saponinas também foi relatada entre os derivados da hederaginina (Voutquenne et al., 2002). Nesse caso, enquanto $\alpha$-hederina e sapindosídeo B são saponinas monodesmosídicas, com glicosilação em C-3 e apresentam forte capacidade hemolítica, a arginina $\mathrm{C}$, uma saponina bidesmosídica com açúcares em C-3 e C-28, também se mostra fortemente hemolítica.

A capacidade do modelo em agrupar seletivamente os diferentes grupamentos substituintes ligados à aglicona foi primeiramente analisada desconsiderando as atividades PHA e $\mathrm{CH} 50 \%$ (Figura 1C). É possível identificar quatro agrupamentos distintos. As variáveis comuns às saponinas de G. max, excetuando a DHSYA, formam o primeiro agrupamento $(1 \mathrm{G})$. Nesse distinguem-se os subs-tituintes C17-met, C22-OH e C21-OH, C22-a, que são comuns às saponinas mono- e bidesmosídicas de soja, respectivamente. Entre C4-met e C4-metox observa-se uma similaridade de $100 \%$, uma vez que ambas variáveis são dependentes. As variáveis C3-d e C22-carb, que são exclusivas de DHSYA e compõem o segundo agrupamento
(2G), também apresentam uma similaridade de 100\%. Os substituintes próprios dos lablabosídeos compõem o agrupamento $3 \mathrm{G}$, no qual se destacam C3-b e C17carbose, já que esses substituintes estão presentes em todos os derivados de $D$. lablab. Os demais substituintes de $3 \mathrm{G}$ correspondem a diversos açúcares que, ao serem diferentes entre as saponinas que compõem esse agrupamento, apresentam menor similaridade. Já o quarto agrupamento $(4 \mathrm{G})$ é composto por substituintes comuns às escinas, como C16-OH, C17-metox, C22-carbmet e C28$\mathrm{OH}$, que por estarem presentes em todos os derivados de A. hippocastanum, apresentam uma similaridade de $100 \%$. Com isso fica comprovada a capacidade do modelo de associar substituintes de acordo com o tipo de saponina selecionada.

A introdução das variáveis PHA e CH50\% (Figura 1D) gera pequenas alterações no dendrograma se comparado ao anterior (Figura 1C). Observa-se que a variável PHA está integrada ao agrupamento $1 \mathrm{G}$, que inclui substituintes comuns às saponinas de G. max, como C22-a, C21-OH e C3-e, refletindo a maior capacidade imunoadjuvante dessas saponinas. A variável $\mathrm{CH} 50 \%$ está associada ao grupamento $4 \mathrm{G}$, que contêm substituintes próprios das escinas, em especial, $\mathrm{C} 16-\mathrm{OH}, \mathrm{C} 17-$ metox, C22-carbmet e $\mathrm{C} 28-\mathrm{OH}$, refletindo a maior capacidade hemolítica dessas saponinas. Os agrupamentos $2 \mathrm{G}$ e $3 \mathrm{G}$ reúnem substituintes de saponinas que apresentam, majoritariamente, moderada atividade imunoadjuvante e baixa atividade hemolítica.

\section{Análise de componentes principais}

Pelo critério da raiz latente, para a análise das saponinas foram extraídos quatro componentes (Figura 2A e 2B) e para a análise dos substituintes foram extraídos onze (Figura 2C e 2D). Esses valores não se alteraram após a introdução de PHA e CH50\% nas análises, indicando que as magnitudes de escala adotadas para ambas as atividades possuem peso similar às variáveis binárias. Ao mesmo tempo, a invariabilidade no número de componentes extraídos indica que a escala utilizada na adequação das variáveis numéricas foi suficientemente sensível, sem provocar distorções no sistema.

Os resultados da análise de componentes principais das saponinas e dos substituintes encontram-se representados na Figura 3.

A capacidade do modelo de correlacionar os diferentes substituintes de acordo com o tipo de saponina de origem foi, novamente, avaliada desconsiderando em um primeiro momento as atividades biológicas PHA e $\mathrm{CH} 50 \%$. Na ausência de atividades biológicas (Figura 3A) observa-se uma forte correlação entre ESC-3A e ESC-3B (quadrante c) atribuída à presença comum de duas metilas em C-4. Esta característica não se repete nas demais escinas, que apresentam uma metila e um $\mathrm{CH}_{2} \mathrm{OH}$ em C-4, dando origem a correlação observada no quadrante d. De 
modo similar, LBL-A e LBL-F apresentam duas metilas em C-4 e por isso aparecem fortemente correlacionadas no quadrante a, segregados dos demais lablabosídeos (LBL-B, LBL-C, LBL-D e LBL-E) que apresentam um $\mathrm{CH}_{2} \mathrm{OH}$ e uma metila em C-4 e que se agregam no quadrante b. Uma forte correlação entre as saponinas derivadas de $G$. max pode ser observada no quadrante d, as quais têm em comum um $\mathrm{CH}_{2} \mathrm{OH}$ e uma metila em C-4. Esses resultados coincidem com os obtidos mediante análise de agrupamentos, onde as diferentes subpopulações dentro de um mesmo agrupamento também são determinadas pelos substituintes em C-4, assim como as correlações observadas nos diferentes quadrantes na análise de componentes principais. Assim, a capacidade do modelo de correlacionar os grupamentos substituintes com as saponinas de origem fica estabelecida.

A introdução de PHA e CH50\% (Figura 3B) leva a um rearranjo nas observações (saponinas), onde as escinas, que são fortemente hemolíticas, estão segregadas das saponinas de $D$. lablab e G. max, que são desprovidas de atividade hemolítica, mas que apresentam atividade imunoadjuvante. Contudo, a sobreposição de vetores neste caso impede o estabelecimento de resultados conclusivos.

$\mathrm{Na}$ análise dos grupamentos substituintes (Figura 3C), a distribuição dos vetores engloba os quatro quadrantes. As variáveis comuns aos lablabosídeos, as escinas e as sojassaponinas estão fortemente correlacionadas nos quadrantes b, c e d, respectivamente. Já a variável C42 met, que se dilui ao longo das estruturas analisadas, não apresenta qualquer correlação direta com as demais variáveis e, portanto, ocupa o quadrante a. As correlações observadas coincidem com os resultados anteriormente obtidos pela análise de agrupamentos, onde os diferentes quadrantes ocupados refletem os agrupamentos formados e os desvios de um vetor em relação ao outro no mesmo quadrante, geralmente, correspondem às diferentes subpopulações observadas na análise de agrupamentos, conjuntamente com o nível de similaridades de uma variável em relação à outra.

Com a inclusão das atividades biológicas PHA e CH50\% na análise, ocorre uma inversão quase completa nos quadrantes (Figura 3D). As variáveis referentes às escinas, anteriormente localizadas no quadrante c (Figura $3 \mathrm{C}$ ), passam ao quadrante $\mathrm{b}$, que é oposto ao anterior. Isso indica que, após a introdução das atividades biológicas, o primeiro componente passa a se relacionar principalmente com as características comuns às escinas. Os módulos dos vetores das variáveis $\mathrm{C} 16-\mathrm{OH}, \mathrm{C} 17$-metox, C22-carbmet, e C28-OH são elevados e no mesmo sentido do vetor da variável $\mathrm{CH} 50 \%$, indicando que a presença desses grupamentos estaria relacionada à capacidade hemolítica. Esta constatação é reforçada pela análise de agrupamentos e pelo estudo realizado por Voutquenne et al. (2002) segundo o qual a presença de uma $\alpha$-hidroxila em C-16, de uma glicosilação em C-3 e de uma carboxila livre em C-28, seriam determinantes para a atividade hemolítica.
Associado a isso, Hostettmann \& Marston (1995), afirmam que a poliidroxilação ou a concentração de grupamentos polares nos anéis D e E promove a atividade hemolítica, o que coincide com os resultados obtidos pelos métodos estatísticos. Por outro lado, os grupamentos C17-met, C3-b e C17-carbose parecem determinantes para a atividade imunoadjuvante, dada a maior similitude entre esses vetores e aquele da variável PHA.

Os substituintes C21-tig e C21-ang exemplificam saponinas aciladas nessa posição. Em ambos os casos, o módulo dos respectivos vetores é discreto e no mesmo sentido da variável $\mathrm{CH} 50 \%$ (figura $3 \mathrm{D}$ ), indicando que a presença do grupamento acila em C-21 favorece a atividade hemolítica das saponinas, conforme anteriormente relatado na literatura (Matsuda et al., 1997).

O sentido oposto, em quase $180^{\circ}$, dos vetores que expressam PHA e $\mathrm{CH} 50 \%$, sugere a existência de uma forte relação de oposição entre as atividades imunoadjuvante e hemolítica, contradizendo a literatura, segundo a qual as atividades imunoadjuvante e hemolítica seriam independentes (Oda et al., 2000). Essa conclusão se origina, sobretudo, do comportamento da saponina QS-21 de Quillaja saponaria, que apresenta elevada capacidade imunoadjuvante e hemolítica, concomitantemente. Contudo, este fato, em um âmbito geral, parece exceção, visto que a tendência observada indica a existência de uma relação de oposição entre ambas as atividades.

A capacidade imunoadjuvante das saponinas parece estar relacionada à presença dos substituintes $\mathrm{C} 22$-a, C21-OH e C3-e, no caso da análise de agrupamentos, e a presença de C17-met, C3-b e C17-carbose, no caso da análise de componentes principais. A associação dessas informações sugere que o número de açúcares ligados a aglicona seria relevante para a atividade imunoadjuvante assim como anteriormente relatado por Oda et al. (2003) para as saponinas de soja, sendo que as bidesmosídicas teriam uma maior atividade imunoadjuvante se comparadas as monodesmosídicas. Contudo, a comparação entre as saponinas monodesmosídicas SYA-1, SYA-2 e SYA-3 de G. max e as saponinas bidesmosídicas de D. lablab, mostra que as primeiras possuem, em sua maioria, uma maior capacidade imunoadjuvante embora sejam monodesmosídicas. Neste caso, a presença de C17met nas sojassaponinas, mas que está ausente nos lablabosídeos, surge como fator de diferenciação. Este fato sugere que o número de açúcares ligados a aglicona está subordinado às características estruturais da aglicona e provavelmente exerce um papel secundário sobre a atividade imunoadjuvante, assim como anteriormente observado para a atividade hemolítica.

Há certo paralelismo, no caso da atividade imunoadjuvante, com o papel inespecífico desempenhado pelo número, natureza e grau de ramificação das cadeias de açúcares na atividade hemolítica. Enquanto que as saikossaponinas possuem atividade hemolítica e essa atividade foi associada ao número de açúcares da cadeia 


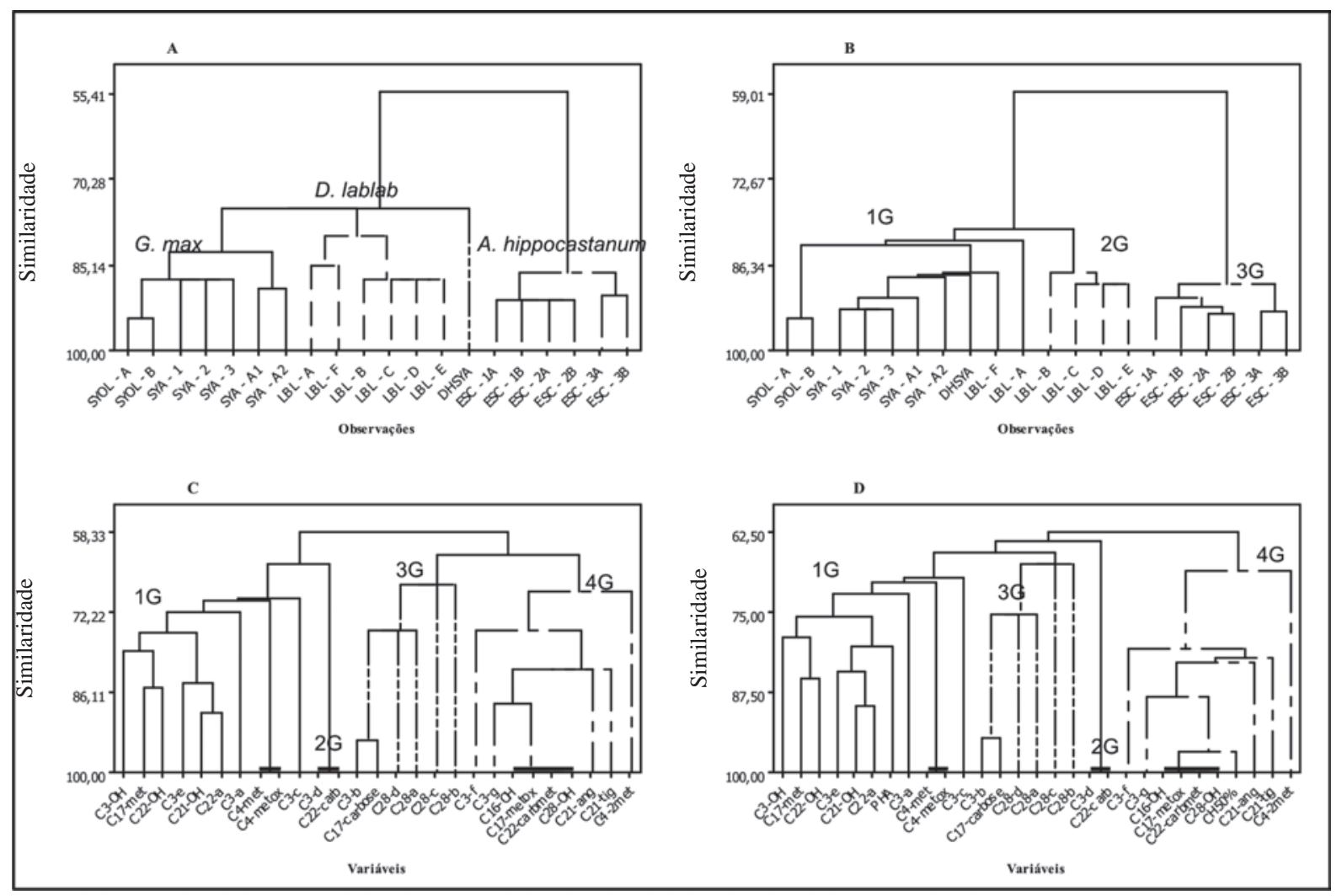

Figura 1. Dendrogramas das saponinas e grupamentos substituintes na ausência (A e C) de atividades biológicas e na presença (B e D) de PHA e $\mathrm{CH} 50 \%$.

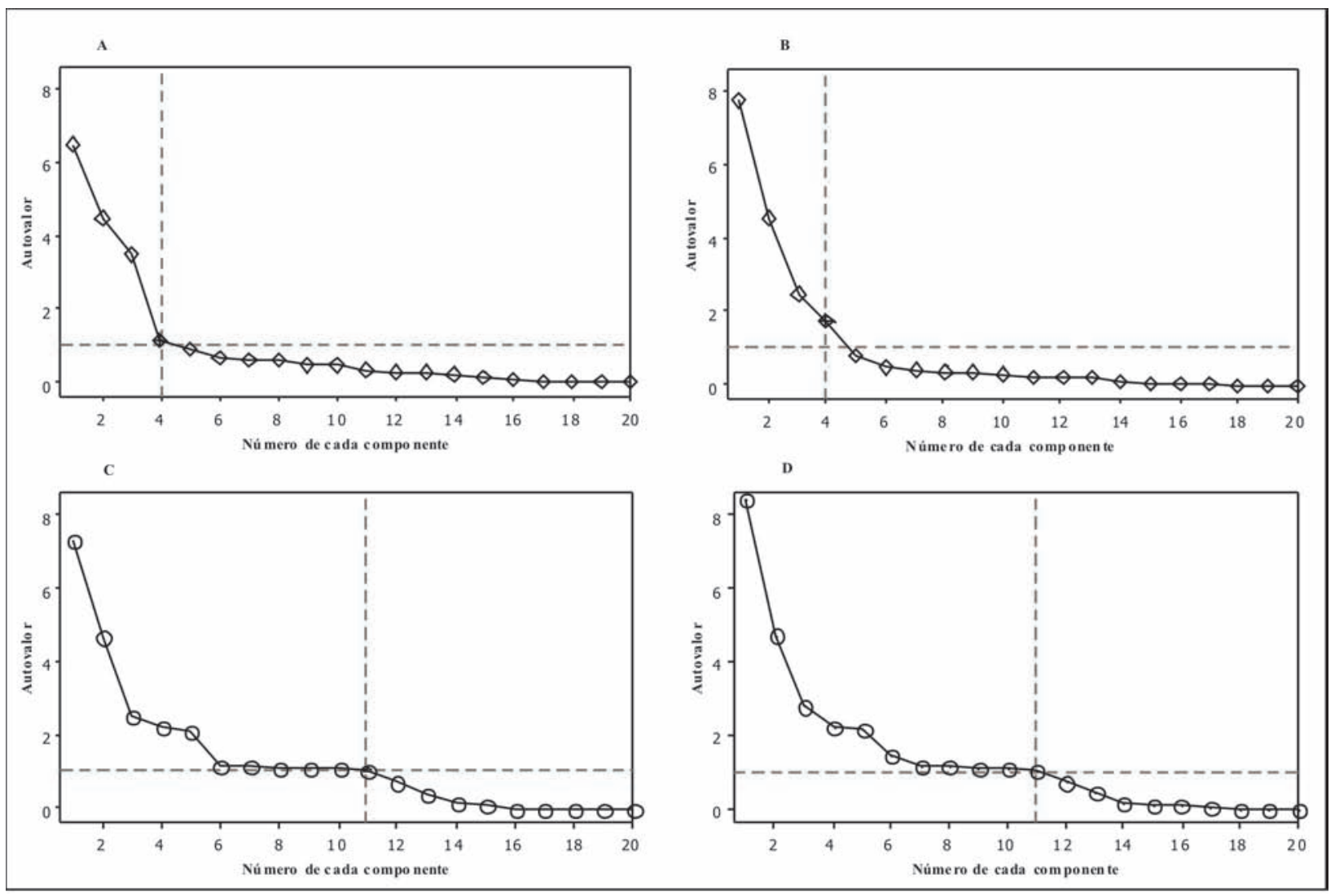

Figura 2. Gráficos de autovalor das saponinas e grupamentos substituintes na ausência (A e C) de atividades biológicas e na presença (B e D) de PHA e CH50\%. 


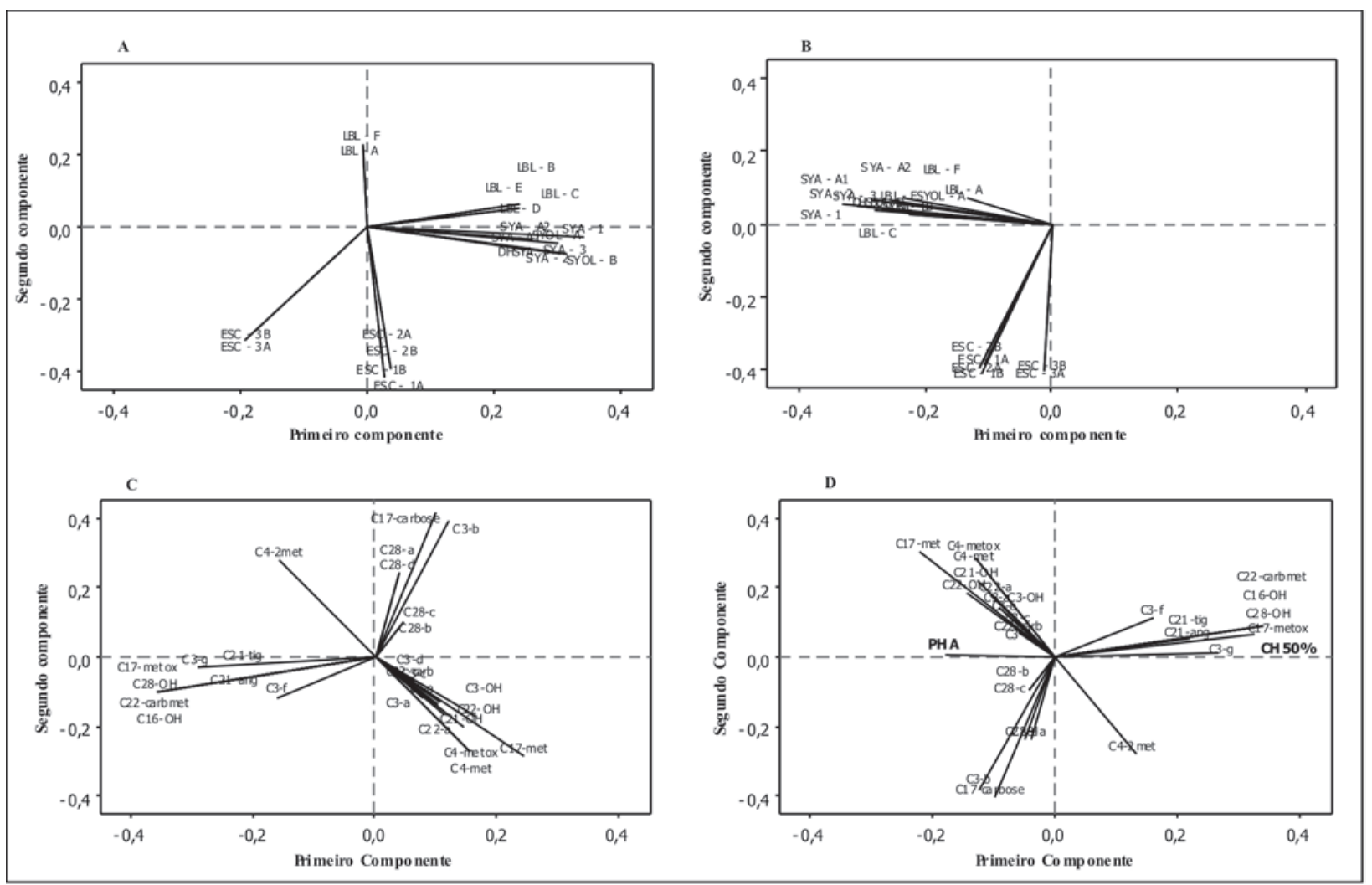

Figura 3. Representação gráfica da análise de componentes principais das saponinas e grupamentos substituintes na ausência (A e C) de atividades biológicas e na presença (B e D) de PHA e CH50\%.

glicosídica (Abe et al., 1978), o aumento e a ramificação da cadeia levam à perda dessa atividade em saponinas derivadas da hederagenina (Voutquenne et al., 2002).

\section{CONCLUSÕES}

De um modo geral pode-se constatar que a atividade imunoadjuvante das saponinas é favorecida pela presença de uma hidroxila em C-21, de uma metila em C-17 e pela presença de dois açúcares ou cadeia de açúcares ligados à aglicona, que parecem exercer um papel secundário. No que tange à atividade hemolítica, esta é promovida acentuadamente pela presença de hidroxila em $\mathrm{C}-16, \mathrm{CH}_{2} \mathrm{OH}$ em C-17, acetila em C-22 e um grupamento acila em C-21. Além disso, observa-se a existência de uma relação de oposição entre as atividades imunoadjuvante e hemolítica para as saponinas analisadas.

Embora algumas relações estruturais relacionadas às atividades hemolítica e imunoadjuvante foram confirmadas aplicando ferramentas estatísticas e podem ser consideradas estabelecidas, o panorama geral permanece ainda incerto em vários aspectos. Possivelmente, uma abordagem específica, focada em agliconas estruturalmente relacionadas possa levar a resultados ainda mais tangíveis e inequívocos.

\section{AGRADECIMENTOS}

Agradecemos ao Conselho Nacional de Desenvolvimento Científico e Tecnológico (CNPq) pelo aporte financeiro para a realização deste trabalho. 


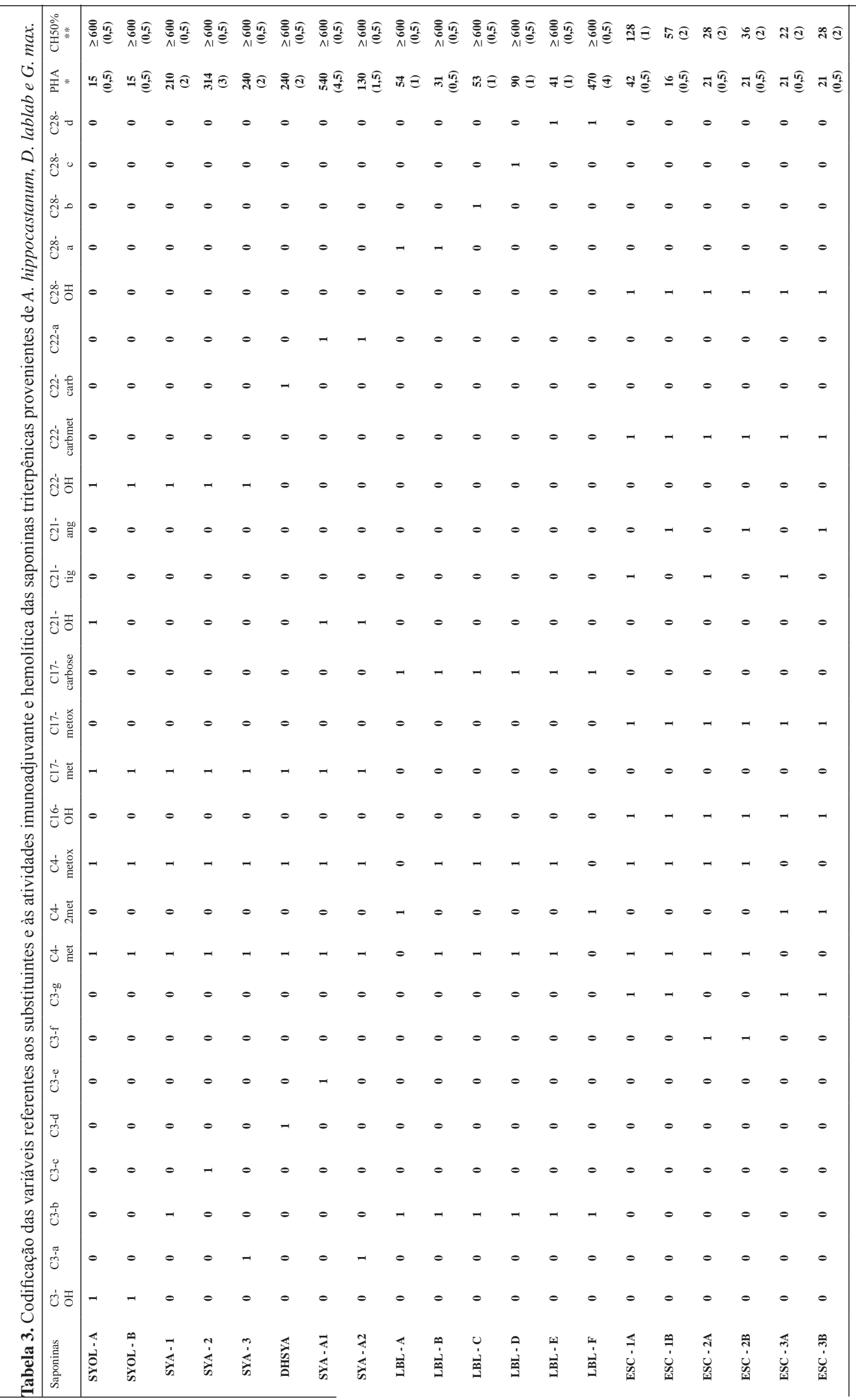




\section{REFERÊNCIAS}

Abe H, Sakaguchi M, Konishi H, Tani T, Arichi S 1978. The effects of saikosaponins on biological membranes. 1. The relationship between the structures of saikosaponins and haemolytic activity. Planta Med 34: 160-166.

Estrada A, Katselis GS, Laarveld B, Barl B 2000. Isolation and evaluation of immunological adjuvant activities of saponins from Polygala senega L. Comp Immunol Microbiol \& Infect Dis 23: 27-43.

Fleck JD, Kauffmann C, Spilki F, Lencina CL, Roehe PM, Gosmann G 2006. Adjuvant activity of Quillaja brasiliensis saponins on the immune responses to bovine herpesvirus type 1 in mice. Vaccine 24: 7129-7134.

Gestetner B, Assa Y, Henis Y, Birk Y, Bondi A 1971. Lucerne saponins. IV. Relationship between their chemical constitution, and heamolytic and antifungal activities. $J$ Sci Food Agric 22: 168- 172.

Hair JF, Anderson RE, Tatham RL, Black WC 2005. Análise multivariada de dados. 5.ed. Porto Alegre: Bookman.

Hase J, Kobashi K, Mitsui K, Namba T, Yoshizaki M, Tomimori T 1981. The structure-hemolysis relationships of oleanolic acid derivatives and inhibition of the saponin-induced hemolysis with sapogenins. J Pharm Dyn 4: 833-837.

Hostettmann K, Marston A 1995. Chemistry and pharmacology of natural products: Saponins. Cambridge: Cambridge University Press.

Kensil CR, Soltysik S, Patel U, Marciani DJ 1992. Structure/ function relationship in adjuvants from Quillaja saponaria Molina. In: Brown F, Chanock RM, Ginsberg HS, Lerner RA (org.). Vaccines 92: Modern approaches to new vaccines including prevention of AIDS. New York: Cold Spring Harbor Laboratory Press, p. 35-40.

Kensil CR, Soltysik S, Wheeler DA, Wu JY 1996. Structure/ function studies on QS-21, a unique immunological adjuvant from Quillaja saponaria. In:Waller G, Yamasaki $\mathrm{K}$ (org.). Saponins used in traditional and modern medicine. New York: Plenum Press, p. 165-172.

Matsuda H, Li Y, Murakami T, Ninomiya K, Yamahara J, Yoshikawa M 1997. Effects of escins Ia, Ib, IIa and IIb from horse chestnut, the seeds of Aesculus hippocastanum L., on acute inflammation in animals. Biol Pharm Bull 20: 1092-1095.

Minitab Inc. 2005. MinitabRelease 14Statistical Software. Minitab Inc., Quality Plaza, State Collage, Pennsylvania.

Oda K, Matsuda H, Murakami T, Katayama S, Ohgitani T, Yoshikawa M 2000. Adjuvant and haemolytic activities of 47 saponins derived from medicinal and food plants. Biol Chem 381: 67-74.

Oda K, Matsuda H, Murakami T, Katayama S, Ohgitani T, Yoshikawa M 2003. Relationship between adjuvant activity and amphipathic structure of soyasaponins. Vaccine 21: 2145-2151.

O’Hagan DT, Mackinchan ML, Singh M 2001. Recent developments in adjuvants for vaccines against infectious diseases. Biomol Eng 18: 69-85.
Schenkel EP, Gosmann G, Athayde ML 2003. Saponinas. In: Simões CMO, Schenkel EP, Gosmann G, Mello JCP, Mentz LA, Petrovick PR (org.). Farmacognosia: da planta ao medicamento. 5.ed. Porto Alegre/Florianópolis: Ed. UFRGS/Ed. UFSC, p. 711-740.

Soltysik S, Wu JY, Recchia J, Wheeler DA, Newman MJ, Coughlin RT, Kensil CR 1995. Structure/function studies of QS-21 adjuvant: assessment of triterpene aldehyde and glucuronic acid roles in adjuvant function. Vaccine 13: 1403-1410.

Sun HX, Qin F, Ye YP 2005. Relationship between haemolytic and adjuvant activity and structure of protopanaxadioltype saponins from the roots of Panax notoginseng. Vaccine 23: 5533-5542.

Sun HX, Pan HJ 2006. Immunological adjuvant effect of Glycyrrhiza uralensis saponins on the immune responses to ovalbumin in mice. Vaccine 24: 1914-1920.

Sun HX 2006. Adjuvant effect of Achyranthes bidentata saponins on specific antibody and cellular response to ovalbumin in mice. Vaccine 24: 3432-3439.

Voutquenne L, Lavaud C, Massiot G, Men-Olivier LL 2002. Structure-activity relationship of heamolytic saponins. Pharm Biol 40: 253-262. 